\title{
CORRECTION
}

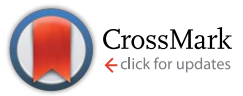

Cite this: J. Mater. Chem. A, 2015, 3, 13577

DOI: $10.1039 / c 5 \operatorname{ta} 90128 f$

www.rsc.org/MaterialsA

\section{Correction: Fibrous and flexible supercapacitors comprising hierarchical nanostructures with carbon spheres and graphene oxide nanosheets}

\author{
Xiong Zhang, ${ }^{a}$ Yuekun Lai, ${ }^{\text {ab }}$ Mingzheng Ge, ${ }^{a}$ Yaxin Zheng, ${ }^{c}$ Ke-Qin Zhang*ab \\ and Zhiqun Lin*d \\ Correction for 'Fibrous and flexible supercapacitors comprising hierarchical nanostructures with carbon \\ spheres and graphene oxide nanosheets' by Xiong Zhang et al., J. Mater. Chem. A, 2015, DOI: 10.1039/ \\ c5ta03252k.
}

The following acknowledgements were omitted from this manuscript.

We gratefully acknowledge the financial support from the National Science Foundation of China under Grant 51073113, 51373110 and 91027039, Natural Science Foundation of Jiangsu Province of China (no. BK20130313), and the Natural Science Foundation of the Jiangsu Higher Education Institutions of China under Grant 10KJA540046. We also acknowledge support from the Priority Academic Program Development of Jiangsu Higher Education Institutions (PAPD), Qing Lan Project for Excellent Scientific and Technological Innovation Team of Jiangsu Province (2012) and Project for Jiangsu Scientific and Technological Innovation Team (2013).

The Royal Society of Chemistry apologises for these errors and any consequent inconvenience to authors and readers.

\footnotetext{
${ }^{a}$ National Engineering Laboratory for Modern Silk, College of Textile and Clothing Engineering, Soochow University, Suzhou, Jiangsu 215123, China. E-mail: kqzhang@suda.edu.cn ${ }^{b}$ Research Center of Cooperative Innovation for Functional Organic, Polymer Material Micro, Soochow University, Nanofabrication, Suzhou, Jiangsu 215123, China 'Department of Electrical and Computer Engineering, University of Waterloo, Ontario, N2L 3G1, Canada

${ }^{d}$ School of Materials Science and Engineering, Georgia Institute of Technology, Atlanta, Georgia 30332, USA. E-mail: zhiqun.lin@mse.gatech.edu
} 